\title{
Establishment of a new representative model of human ovarian cancer in mice
}

Jianjun Zhang ${ }^{1,3,5}$, Xinlian Chen ${ }^{1,4,5}$, Gang Shi ${ }^{3}$, Xiaoyan Xie ${ }^{1,4,5}$, Hongqian Liu ${ }^{1,4,5}$, Xuemei Zhang ${ }^{1,4,5}$, Yi Lai ${ }^{4}$, Yan Zuo ${ }^{3}$, Zhong Chen ${ }^{6^{*}}$, Shanling Liu ${ }^{2,3,4,5^{*}}$ and He Wang ${ }^{1,3,4,5^{*}}$

\begin{abstract}
Background: Intraperitoneal (i.p.) models that accurately mimic the feature behavior of human ovarian cancer are required to investigate the pathology and therapeutics of the disease. However, established i.p. models which are well-characterized and reliable are few. The purposes of this study are to establish a representative mice i.p. model of the disease and to analyze the consequent pathology.

Methods: Fresh tumor cells fiom the ascites of patient were injected into female NOD/SCID mice intraperitoneally. Histology, Cytogenetic, immunohistochemistry,tumor markers of CA125,AFP, CA-199 and CEA were used to analyze the model.

Results: The mice developed marked abdominal distention within 6 months after inoculated with tumor cells from a patient with epithelial ovarian carcinoma. The mice developed clinically evident intraperitoneal tumors and massive ascites containing numerous tumor cells in clumps. CA125 level in our model was high in both serum and ascites supernatants, while levels of other tumor markers, such as AFP, CA-199 and CEA, were normal. Cytogenetic analysis and immunohistochemical staining confirmed its characteristics resembling human epithelial ovarian tumor.
\end{abstract}

Conclusions: The model described in this paper accurately mimics the features of ovarian tumor, which may be useful for evaluation of new therapeutics.

Keywords: Human, Ovary carcinoma, Animal model, CA125, Intraperitoneal

\section{Background}

Ovarian cancer is the leading cause of death among gynecologic tumors with a death toll up to 13850 in the USA by 2010 [1]. Epithelial ovarian cancer (EOC) accounts for over $90 \%$ of all ovarian malignancies. Its high mortality is attributable to the fact that $75 \%$ of the patients are not diagnosed until the advanced stage. Although the majority of the patients respond to initial chemotherapy after a primary debulking surgery, most eventually experience recurrence as they become chemoresistant [2]. Research into

\footnotetext{
* Correspondence: z.chen2000@yahoo.cn; sunny630@126.com; wanghe_cd@ 126.com

${ }^{6}$ Division of Medical Genetics, University of Utah School of Medicine, Salt Lake City, Utah 84132, USA

${ }^{2}$ Laboratory of Cell and Gene Therapy, West China Institute of Maternal and Child Health, West China Second University Hospital, Sichuan University, Chengdu 610041, P. R. China

${ }^{1}$ Laboratory of Genetics, West China Institute of Maternal and Child Health, West China Second University Hospital, Sichuan University, Chengdu 610041, P. R. China

Full list of author information is available at the end of the article
}

the development of well-characterized and reliable models is crucial for evaluating efficacy of novel therapeutics, which may help improve patient survival.

In developing an in vivo model of human ovarian neoplasia, it is critical to ensure that the model mimics the behavior of ovarian tumor in patient accurately. Research teams have attempted relevant models employing subcutaneous (s.c.) and intraperitoneal (i.p.) xenografts in immunodeficient mice [3-16], where only the i.p. models were in line with the clinical manifestations in the advanced stage given the carcinomatosis in the peritoneal cavity with large volumes of ascites. Further more, such models seemed clinically useful in demonstrating efficacy of the intraperitoneal therapies being tested, which was hardly the case with the s.c. models. Most of the models commonly used in ovarian cancer research are based on established cell lines. However, it is found that, compared with the cell lines, only xenografts established directly from fresh human ovarian tumor tissues

\section{Biomed Central}

(c) 2013 Zhang et al.; licensee BioMed Central Ltd. This is an Open Access article distributed under the terms of the Creative Commons Attribution License (http://creativecommons.org/licenses/by/2.0), which permits unrestricted use, distribution, and reproduction in any medium, provided the original work is properly cited. 
could match the original tumors in terms of antigen gene expression [12], as may be attributed to the fact that the cell lines could have changed their protein expression patterns and lost the heterogenetic characteristics of human cancer through long-term in vitro culturing.

Serum tumor markers also seem useful in the management of several types of ovarian tumor. For example, CA125, measurable in the serum, is routinely used as a diagnostic biomarker of ovarian cancer in clinical settings, which, however, due to its low sensitivity in the early stage, tends to make better sense for monitoring tumor progression and response to therapy. Its use for evaluating therapeutic efficacy has been attempted in a few studies $[17,18]$.

In our study, we employed the NOD/SCID mice and fresh tumor cells, and established a novel reproducible xenograft model of ovarian cancer, which is transplantable and is characterized by its close mimic of the progressive massive ascites, extensive intra-abdominal carcinomatosis, and elevated CA125 levels in both blood and ascites.

\section{Methods}

\section{Clinical characteristics}

The patient was a 53-year-old post-menopausal woman presenting with a 2-month history of abdominal distention with a mass in the right adnexa. Cytological study of the ascites indicated presence of adenocarcinoma cells, with a serum CA125 level of 1364 U. She received intraperitoneal chemotherapy with Thiotepa twice $\left(1^{\text {st }}\right.$ : $30 \mathrm{mg}, 2^{\text {nd }}: 20 \mathrm{mg}$ ) before surgery (cyto-reductive surgery, extrafascial hysterectomy, bilateral adnexectomy, omentectomy, appendectomy). Poorly differentiated serous adenocarcinomas of the ovary (stage FIGO IIIC) were pathologically confirmed. Informed consent was acquired from the patient.

\section{Animals}

Four- to six-week-old female NOD/SCID mice (purchased from Beijing HFK Bio-Technology Co. Ltd., Beijing, China) were housed in sterile micro-isolators (5 mice per cage). Feeding and water were given ad libitum. All procedures were performed under sterile conditions in a laminar flow hood. The animals were monitored daily for general health status. All animal experiments described in this study were approved by the Institutional Animal Care and Use Committee of Sichuan University.

\section{Heterotransplantation and in vivo Passaging}

A tumor specimen (ascites) was collected and sterilely transported on ice to the laboratory. The ascites was centrifuged at $300 \mathrm{G}$ for $5 \mathrm{~min}$. The cell pellet was immediately intraperitoneally injected into the NOD/SCID mice. The number of tumor cells from the patient inoculated into the two mice was $5 \times 10 \mathrm{E} 7$.

When the animals had marked abdominal distensions, their ascites was collected from the tumor-bearing mice, and directly injected into the next mice. The mice were then monitored daily for general health status as well as the degree of abdominal extension before another passaging. Xenografts were established within 6 months after heterotransplantation in both mice.As passaging continued, the number of tumor cells we used was $1 \times 10 \mathrm{E} 7$. The peritoneal cavity of each animal was examined. Tissues and organs suspected of being affected by tumor were eventually harvested for histological examinations.

\section{Freezing storage of xenograft tumor}

After the animals were sacrificed, ascites was collected and centrifuged at $300 \mathrm{G}$ for $5 \mathrm{~min}$. The pellet was suspended in cold freezing media, consisting of DMEM with $8 \%$ DMSO and $20 \%$ fetal bovine serum (FBS). The cells were then transferred to liquid nitrogen for longterm storage. For retransplantation, the tumor suspension underwent a rapid thaw at $37^{\circ} \mathrm{C}$, and was diluted in $10 \mathrm{ml}$ of DMEM without FBS. Then it was centrifuged, and intraperitoneally injected into the mice.

\section{Immunohistochemistry}

Tumors in the peritoneal cavity were fixed in $4 \%$ neutral buffered formalin, and processed for histological examination. The fixed samples were embedded in paraffin, $5 \mu$-thick sections were cut, and H-E staining was applied. Cell suspensions from ascites were cyto-centrifuged, and smear was fixed with cold acetone. Endogenous peroxidase activity was then blocked with $3 \%$ hydrogen peroxide in PBS for $10 \mathrm{~min}$. The samples were subsequently rinsed with $\mathrm{PBS}$ for $5 \mathrm{~min}$. All tissues were blocked with BSA for $20 \mathrm{~min}$ at room temperature. Primary monoclonal antibodies to vimentin (dilution 1:300), pan-cytokeatin (dilution 1:400), EMA (dilution 1:500), P53 (dilution 1:300), MMP-2 (dilution 1:300) (all supplied by Wuhan Boster Bio-Engineering Co. Ltd., Wuhan, China), and CA125 (dilution 1:400) (supplied by Beijing Zhongshan Golden Bridge Biotechnology Co. Ltd., Beijing, China) were used. Sections were incubated with the antibodies overnight at $4^{\circ} \mathrm{C}$ following three 5min washes with PBS, then incubated with biotinylated secondary antibody at $37^{\circ} \mathrm{C}$ for $20 \mathrm{~min}$, and finally incubated with streptavidin-biotin-horse radish peroxidase complex at $37^{\circ} \mathrm{C}$ for $20 \mathrm{~min}$. The sections were then developed with diaminobenzidine (DAB) as a substrate. Cellular nuclei were counterstained with hematoxylin. All operations followed the manufacturers' recommendations. Absent primary antibody during processing was used as blank control. Immunostaining was semiquantitative as described [19]. 


\section{Analysis of tumor marker in blood and Ascites}

Whole blood and ascites specimens were collected from the tumor-bearing mice, and centrifuged for $10 \mathrm{~min}$. The supernatants were isolated and stored at $-20^{\circ} \mathrm{C}$ for subsequent analysis. Levels of CA125, CA199, AFP and CEA were determined.

\section{Cytogenetics}

Ascites specimens were collected and centrifuged. Cell pellet was resuspended in DMEM supplemented with $10 \%$ heat-inactivated fetal calf serum (FCS), and exposed to $0.5 \mu \mathrm{g} / \mathrm{ml}$ demecolcine (Colcemid) for 2 hours. Cytogenetic analysis was accomplished by G-banding using a conventional Giemsa staining protocol [20]. Briefly, tumor cells were harvested and incubated in $75 \mathrm{mM}$ potassium chloride at $37^{\circ} \mathrm{C}$. Then they were fixed with $25 \%$ acetic acid in anhydrous methanol twice. The slides were trypsinized and stained with $10 \%$ Giemsa, and 35 metaphase spreads were karyotyped.

\section{Results}

\section{Growth of human ovarian tumors in scid mice}

Tumor cells in the ascites from the patient with poorly differentiated ovary papillary serous adenocarcinoma were injected into the peritoneal cavities of female NOD/SCID mice. Xenografts were established within 6 months after heterotransplantation. As passaging continued, the time to achieve a clinically evident disease decreased to 1-2 months. All mice eventually developed visible abdominal swelling (Figure 1A) and yellowish pale ascites (Figure 1B). The volume of ascites was generally 4 to $8 \mathrm{ml}$ per mouse. Abundant cells of clinical
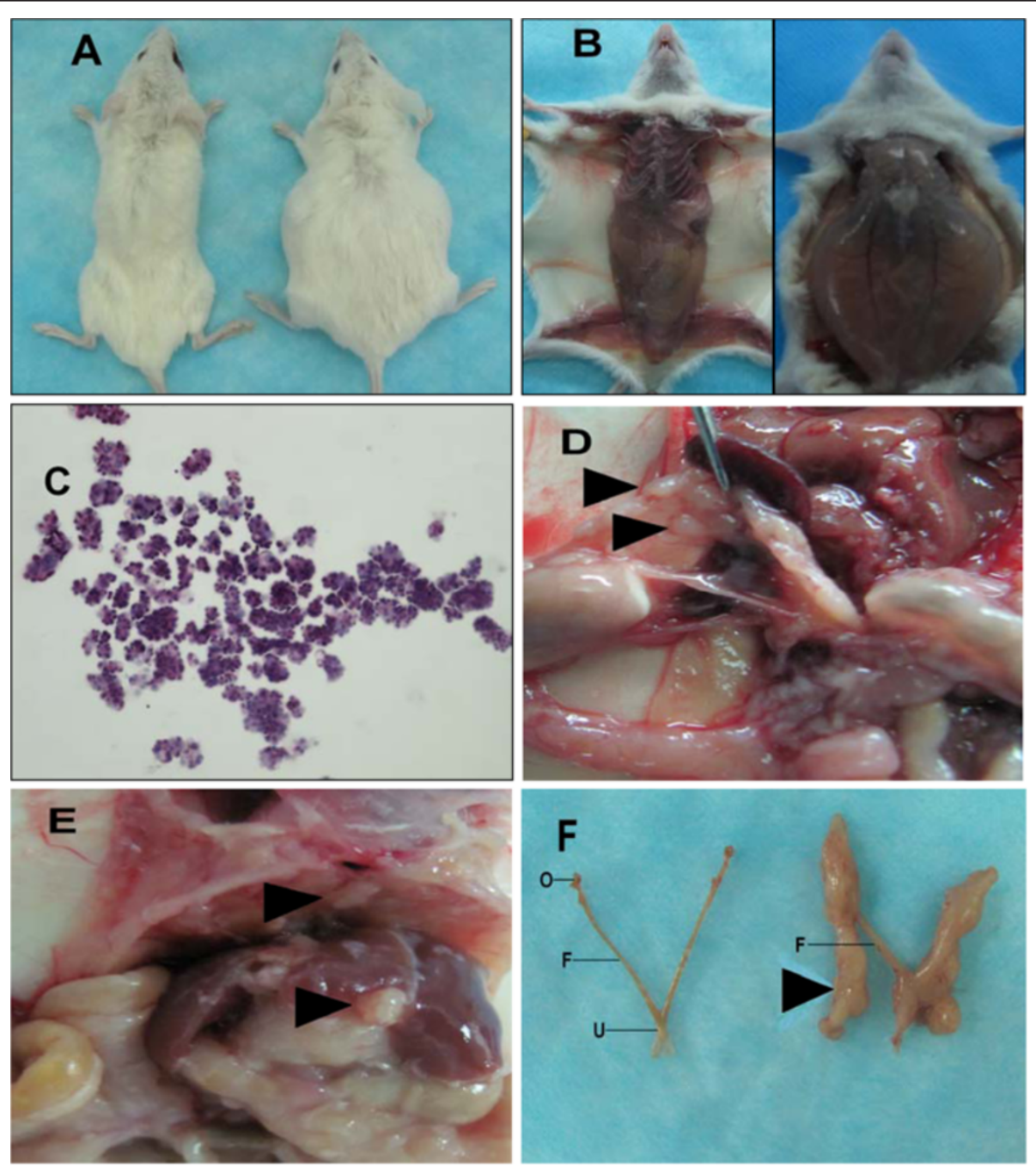

Figure 1 (A) Female NOD/SCID mice with abdominal distention after injection of ascites tumor cells. Left image is non-injected control; (B) Presence of milky ascites in mice; (C) Large, crowded clumps of cells in ascites; (D) Mesentery invaded by tumor tubercles; (E) Subdiaphragmatic tumor deposits and deposits on liver surface; (F) Reproductive system affected by tumor. Left image is non-injected control. Arrowhead indicates metastatic tumor. O-ovary; S-fallopian tube; U-uterus. 
resemblance were seen individually or in clusters on cytological examination of the ascites (Figure 1C). The malignant features of the cells were confirmed by high nuclear-cytoplasmic ratio, nuclear multiforme and predominant nucleoli; and these histological characteristics were documented in different mouse generations. The mean viable tumor cell yield from ascites was approximately $1.5 \times 10^{9}$ cells per mouse. The malignant cells in the ascites could be reproducibly maintained and directly passaged to subsequent hosts. The cells have been serially transplanted 8 times to date. This model may be reproduced using
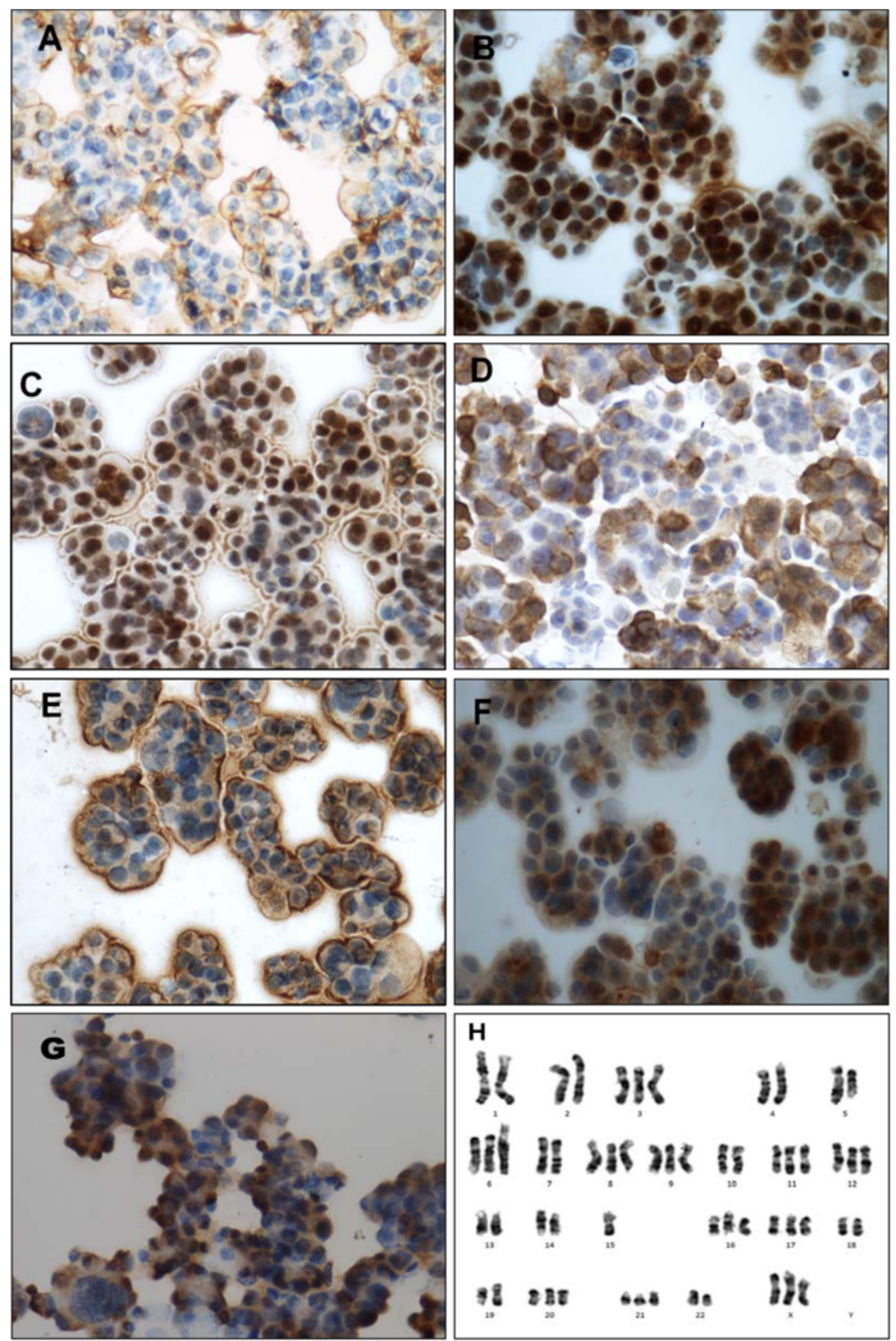

Figure 2 Immunohistochemical staining and Karyotyping profile of xenograft tumors from ascites of the sixth generation. (A) EMA (B) PCNA; (C) P53; (D) Vimentin; (E) CA125; (F) Pan-cytokeatin; (G) MMP-2 (x400); (H) Karyotyping profile of malignant cells from ascites. Number of chromosomes generally between 54 and 60, mostly triploidy with structural abnormalities. 
our cryopreserved tumor cells in liquid nitrogen free of histological changes.

\section{Metastatic pattern}

The metastatic pattern was investigated, where similar results were noted in different generations. In the advanced stage of this model, the mesentery was most frequently invaded by tumor tubercles (Figure 1D). Deposits of tumor cells were found on the surfaces of peritoneal organs, such as the sub-diaphragmatic and liver linings, which replicated the initial mice (Figure 1E). The reproductive system was severely affected by tumor cells (Figure 1F). Severe unilateral or bilateral ovarian pathogenesis was found in $75 \%$ of the animals in our model. In most cases, the histomorphology and cytology of ovary changed as the condition developed from crowding and displacement to complete invasion. All suspicious areas were biopsied. No evidence of extraperitoneal spread was noted.

\section{Cytogenetics}

Human female origin of the established xenograft model was confirmed by cytogenetic analysis. Thirty-five banded metaphases were analyzed. Chromosome number ranged from 54 to 60 (median: 58), mainly trisomy (Figure $2 \mathrm{H}$ ).

\section{Immunohistochemistry}

To analyze the histopathology of the tumor cells in the ascites, immunohistochemical staining was performed to determine expression of epithelial membrane antigen (EMA), Pan-cytokeatin, vimentin (VIM), PCNA, P53, CA125 and MMP-2. The results are given in Table 1 and Figure 2. It's found that there is no significant changes in comparison with the first generation (the pictures were showed as Additional file 1: Figure S3).

\section{Tumor makers in serum and ascites}

In analyzing the CA125, CA199, CEA and AFP levels in serum and ascites, we found that only the CA125 level

Table 1 Immunohistochemical markers of heterotransplanted tumor cells from ascites of the sixth generation

\begin{tabular}{lll}
\hline Marker & Result & Method \\
\hline CA125 & ++ & $\mathrm{HC}{ }^{*}$ \\
EMA & ++ & $\mathrm{HC}$ \\
Pan-Cytokeratin & ++ & $\mathrm{HC}$ \\
VIM & ++ & $\mathrm{HC}$ \\
PCNA & ++++ & $\mathrm{HC}$ \\
P53 & +++ & $\mathrm{HC}$ \\
MMP-2 & ++ & $\mathrm{HC}$ \\
\hline
\end{tabular}

* IHC: Immunohistochemical staining. rose in both serum and ascites in the tumor-laden mice. The serous CA125 level ranged 293.4-1024.4U/ml. Supernatant of the malignant ascites was found to contain 484.2-3800U of CA125 per $\mathrm{ml}$. The normal serous CA125 level $(<11 \mathrm{U} / \mathrm{ml})$ was documented in all healthy control mice.

\section{Discussion}

We established a reproducible murine xenograft model of ovarian carcinoma employing tumor cells harvested from the ascites of an ovarian cancer patient, where the cells may be reproducibly maintained and directly passaged to subsequent hosts. This model requires no selective methods before intraperitoneal growth such as enzymatic digestion, selective anchorage-independent growth, and subcutaneous inoculation $[7,12,15,16]$. As a result, the unwanted implications of the manipulations were avoided.

The tumor cells from the ascites were hyperchromatic for CA125 antigen, Pan-cytokcratin and epithelial antigen, which confirmed it to be epithelial differentiation [8]. MMP-2 is believed to be involved in two aspects of the ovarian cancer spread process: It helps tumor cells penetrate the basement membrane of the ovary to invade the stroma [21], and may enable the tumor cells to detach from the epithelial surface and migrate into the peritoneal cavity [22,23]. It is reported that the rate of MMP2 expression in ovarian cancer is high and irrelevant with either clinical staging or histological typing [24]. One possible explanation was the grave malignance of ovarian cancer. In our model, we also noted tumor cells with high MMP-2 expression. P53 mutation occurs early in the progression of ovarian cancer [25], which is found in some $50 \%$ or more of advanced serous adenocarcinomas while rarely noted in earlier stages [26-28]. In this model, tumor cells show intense expression of p53, consistent with the previous reports.

We examined the transplanted tumors in ascites at different passages, and found that the characteristics did not change from one generation to another. As a result, this may offer an unlimited in vivo supply of stable tumor cells, which may be of great value for ensuring reproducibility and reliability of research results. In the future, we may establish similar models of other ovarian cancer subtypes for furthering our knowledge on the disease.

In this model, we examined the CA125, CA199, CEA and AFP levels in blood and ascites, only to find that the CA125 level alone rose in the tumor-bearing mice. This makes a possibility where CA125 may be used as a biomarker in the model, similar to its clinical application, for prognosis prediction and therapeutic evaluation in future studies. 


\section{Conclusions}

This novel model of ovarian cancer, which mimics human carcinosis accurately, appears simple and reproducible. It may also be instrumental for culturing ovarian carcinomas in vivo, hence as an unlimited source of ovarian cancer cells for research purposes. Great merit is seen in our model for evaluating new therapeutics given its CA125 level matching the human situation closely.

\section{Additional file}

Additional file 1: Immunohistochemical staining a of xenograft tumors from ascites of the first generation. (A1) EMA; (B1) PCNA; (C1) P53; (D1) Vimentin; (E1) CA125; (F1) Pan-cytokeatin; (G1) MMP-2 (x400)

\section{Competing interests}

We have no proprietary, financial, professional or other personal interest of any kind in any product, service and/or company that could be construed as influencing the position presented in the manuscript entitled:Establishment of a new representative model of human ovarian cancer in mice.

\section{Authors' contributions}

JJ Z carried out the heterotransplantation and in vivo passaging of the model, participated in in its design and drafted the manuscript. $H Q L, X M Z$, $X Y X$ carried out the immunoassays. $X L C, Y L$ participated in the Cytogenetics study. GS, YZ participated in the collection and transport of clinical specimens and heterotransplantation. ZC ,SL L, HW conceived of the study, and participated in its design and coordination and helped to draft the manuscript. All authors read and approved the final manuscript.

\section{Acknowledgements}

This work was supported by National Natural Science Foundation of China grants $(30973461,81270660,30772323)$ awarded to HW and SL L, the Science Grant from Science and Technology Department of Sichuan Province, China (2008SZ0018, 2012SZ0136) awarded to SL L. This work was also partly supported by Beijing Yi Ke Wan Yun Technology Co. Ltd.

\section{Author details}

${ }^{1}$ Laboratory of Genetics, West China Institute of Maternal and Child Health, West China Second University Hospital, Sichuan University, Chengdu 610041, P. R. China. 'Laboratory of Cell and Gene Therapy, West China Institute of Maternal and Child Health, West China Second University Hospital, Sichuan University, Chengdu 610041, P. R. China. ${ }^{3}$ Department of Obstetrics and Gynecology, West China Second University Hospital, Sichuan University, Chengdu 610041, P. R. China. " Prenatal Diagnosis Center of Sichuan Province, West China Second University Hospital, Sichuan University, Chengdu 610041, P. R. China. ${ }^{5}$ Key Laboratory of Obstetrics, Gynecology, Pediatric Diseases and Birth Defects of Ministry of Education, Chengdu 610041, P. R. China. ${ }^{6}$ Division of Medical Genetics, University of Utah School of Medicine, Salt Lake City, Utah 84132, USA.

Received: 8 November 2012 Accepted: 1 February 2013

Published: 6 February 2013

\section{References}

1. Jemal A, Siegel R, Xu JQ, Ward E: Cancer statistics, 2010. CA-Cancer J Clin 2010, 60:277-300.

2. Deraco M, Baratti D, Laterza B, Balestra MR, Mingrone E, Macrì A, Virzì S, Puccio F, Ravenda PS, Kusamura S: Advanced cytoreduction as surgical standard of care and hyperthermic intraperitoneal chemotherapy as promising treatment in epithelial ovarian cancer. Eur J Surg Oncol 2011, 37:4-9.

3. Shaw TJ, Senterman MK, Dawson K, Crane CA, Vanderhyden BC: Characterization of Intraperitoneal, Orthotopic, and Metastatic Xenograft Models of Human Ovarian Cancer. Mol Ther 2004, 10:1032-1042.
4. Elkas JC, Baldwin RL, Pegram M, Tseng Y, Slamon D, Karlan BY: A human ovarian carcinoma murine xenograft model useful for preclinical trials. Gynecol Oncol 2002, 87:200-206.

5. Verschraegen CF, Hu W, Du Y, Mendoza J, Early J, Deavers M, Freedman RS, Bast RC Jr, Kudelka AP, Kavanagh JJ, Giovanella BC: Establishment and characterization of cancer cell cultures and xenografts derived from primary or metastatic Mullerian cancers. Clin Cancer Res 2003, 9:845-852.

6. Sallinen $H$, Anttila M, Narvainen J, Ordén MR, Ropponen K, Kosma VM, Heinonen S, Yla-Herttuala S: A highly reproducible xenograft model for human ovarian carcinoma and application of MRI and ultrasound in longitudinal follow-up. Gynecol Oncol 2006, 103:315-320.

7. Massazza G, Tomasoni A, Lucchini V, Allavena P, Erba E, Colombo N, Mantovani A, D'Incalci M, Mangioni G, Giavazzi R: Intraperitoneal and subcutaneous xenografts of human ovarian carcinoma in nude mice and their potential in experimental therapy. Int J Cancer 1989, 44:494-500.

8. Molpus KL, Koelliker D, Atkins L, Kato DT, Buczek-Thomas J, Fuller AF, Hasan $\mathrm{T}$ : Characterization of a xenograft model of human ovarian carcinoma which produces intraperitoneal carcinomatosis and metastases. Int J Cancer 1996, 67:588-595.

9. Lin XJ, Chen XC, Wang L, Wei YQ, Kan B, Wen YJ, He X, Zhao X: Dynamic progression of an intraperitoneal xenograft model of human ovarian cancer and its potential for preclinical trials. J Exp Clin Cancer Res 2007, 26:467-474.

10. Hamilton TC, Young RC, Louie KG, Behrens BC, McKoy WM, Grotzinger KR, Ozols RF: Characterization of a xenograft model of human ovarian carcinoma which produces ascites and intraabdominal carcinomatosis in mice. Cancer Res 1984, 44:5286-5290.

11. Walker W, Gallagher G: The development of a novel immunotherapy model of human ovarian cancer in human PBL-severe combined immunodeficient (SCID) mice. Clin Exp Immunol 1995, 101:494-501.

12. Ward BG, Wallace K, Shepherd JH, Balkwill FR: Intraperitoneal xenografts of human epithelial ovarian cancer in nude mice. Cancer Res 1987, 47:2662-2667

13. Davy M, Mossige J, Johannesson J: Heterologous growth of human ovarian cancer: a new in vivo testing system. Acta Obstet Gynecol Scand 1977, 56:55-59.

14. Kullander S, Rausing A, Trope C: Human ovarian tumor heterotransplanted to"Nude"mice. Acta Obstet Gynecol Scand 1978, 57:149-159.

15. Schumacher U, Adam E, Horny HP, Dietl J: Transplantation of a human ovarian cystadenocarcinoma into severe combined immunodeficient (SCID) mice-formation of metastases without significant alteration of the tumor cell phenotype. Int J Exp Pathol 1996, 77:219-227.

16. Schumacher U, Adam E, Dietl J, Horny HP: Immunophenotype of human ovarian malignancies (cystadenocarcinomata and mixed mullerian tumor) established in SCID mice. Exp Mol Pathol 1997, 64:103-113.

17. Kraeber-Bodere F, Mishra A, Thedrez P, Faivre-Chauvet A, Bardies M, Imai S, Boterff J, Chatal JF: Pharmacokinetics and biodistribution of samarium153-labelled OC125 antibody coupled to CITCDTPA in a xenograft model of ovarian cancer. Eur J Nucl Med 1996, 23:560-567.

18. Burbridge MF, Kraus-Berthier L, Naze M, Pierre A, Atassi G, Guilbaud N: Biological and pharmacological characterisation of three models of humanhe ovarian carcinoma established in nude mice: use of the CA125 tumour marker to predict antitumour activity. Int J Oncol 1999, 15:1155-1162.

19. Axiotis CA, Monteagudo C, Merino MJ, LaPorte N, Neumann RD: Immunohistochemical detection of P-glycoprotein in endometrial adenocarcinoma. Am J Pathol 1991, 138:799-806.

20. Seabright M: A rapid banding technique for human chromosomes. Lancet 1971, 298:971-972.

21. Kuwashima Y, Uehara T, Kurosumi M, Kishi K, Shiromizu K, Matsuzawa M, Takayama S: Basement membrane status in undifferenciated carcinomas of the ovary. Immunohistochemical distribution of type IV collagen and laminin. Eur J Gynecol Oncol 1995, 16:81-186.

22. Wu XH, Li HL, Kang L, Li L, Wang WP, Shan BE: Activated matrix metalloproteinase-2-A potential marker of prognosis for epithelial ovarian cancer. Gynecol Oncol 2002, 84:126-134.

23. Birkedal-Hansen $\mathrm{H}$ : Proteolytic remodeling of extracellular matrix. Curr Opin Cell Biol 1995, 7:728-735.

24. Suzuki M, Iwasaki M, Sugio A, Hishiya A, Tanaka R, Endo T, Takayama S, Saito T: BAG3 (BCL2-associated athanogene 3) interacts with MMP-2 to positively regulate invasion by ovarian carcinoma cells. Cancer Lett 2011, 303:65-71. 
25. Leitao MM, Soslow TA, Baergen RN, Olvera N, Arroyo C, Boyd J: Mutation and expression of the TP53 gene in early stage epithelial ovarian carcinoma. Gynecol Oncol 2004, 93:301-306.

26. Kupryjanczyk J, Thor AD, Beauchamp R, Merritt V, Edgerton SM, Bell DA, Yandell DW: P53 gene mutation and protein accumulation in human ovarian cancer. Proc Natl Acad Sci USA 1993, 90:4961-4965.

27. Wen WH, Reles $A$, Runnebaum IB, Sullivan-Halley J, Bernstein L, Jones LA, Felix JC, Kreienberg R, El-Naggar A, Press MF: P53 mutations and expression in ovarian cancers: collelation with overall survival. Int J Gynecol Pathol 1999, 18:29-31.

28. Chan WY, Cheung KK, Schorge JO, Huang LW, Welch WR, Bell DA, Berkowitz RS, Mok SC: BCl-2 and p53 protein expression, apoptosis, and p53 mutation in human epithelial ovarian cancers. Am J Pathol 2000, 156:409-417.

doi:10.1186/1757-2215-6-9

Cite this article as: Zhang et al: Establishment of a new representative

model of human ovarian cancer in mice. Journal of Ovarian Research 2013 6:9.

\section{Submit your next manuscript to BioMed Central and take full advantage of:}

- Convenient online submission

- Thorough peer review

- No space constraints or color figure charges

- Immediate publication on acceptance

- Inclusion in PubMed, CAS, Scopus and Google Scholar

- Research which is freely available for redistribution 